\title{
TRANSCENDENTAL ENTIRE FUNCTIONS MAPPING EVERY ALGEBRAIC NUMBER FIELD INTO ITSELF
}

\author{
A. J. VAN DER POORTEN
}

(Received 27 June 1966)

1

T. Schneider [1] has shown that a transcendental function with a limited rate of growth cannot assume algebraic values at too many algebraic points. It is not clear however whether a transcendental function may assume algebraic values at all algebraic points in an open set in its domain of analyticity. We show, using a method of B. H. Neumann and R. Rado [2] that this can be the case. Indeed we construct transcendental entire functions which, together with all their derivatives, assume, at every point in every algebraic number field, values in that field.

\section{2}

Let

$$
Q: q_{1}(z), q_{2}(z), \cdots
$$

be a sequence of all non-constant polynomials with rational integer coefficients and let $q_{n}(z)$ be of degree $d_{n}$. Choose positive integers $k_{1}, k_{2}, \cdots$ such that

$$
k_{n}+d_{1}+d_{2}+\cdots d_{n}<k_{n+1}
$$

and then positive rational numbers $a_{1}, a_{2}, \cdots$ such that

$$
a_{n} \max _{|z| \leqq n}\left|z^{k_{n}} q_{1}(z) q_{2}(z) \cdots q_{n}(z)\right|<2^{-n}(n=1,2, \cdots) .
$$

Then the series

$$
\sum_{n=1}^{\infty} a_{n} z^{k_{n}} q_{1}(z) q_{2}(z) \cdots q_{n}(z)
$$

converges uniformly in every circle and its sum $f(z)$ has the required properties.

We note firstly that we may speak of a sequence $Q$ of all non-constant polynomials with rational integer coefficients by virtue of the countability of the set of all such polynomials, and secondly that a choice of rational 
numbers $a_{1}, a_{2}, \cdots$ is always possible since the polynomial

$$
p_{n}(z)=z^{k_{n}} q_{1}(z) q_{2}(z) \cdots q_{n}(z)
$$

is certainly bounded in the circle $|z| \leqq n$. $(n=1,2, \cdots)$. By (2) the series

$$
\sum_{n=1}^{\infty} a_{n} z^{k_{n}} q_{1}(z) q_{2}(z) \cdots q_{n}(z)=\sum_{n=1}^{\infty} a_{n} p_{n}(z)
$$

converges uniformly in every circle, whence its sum $f(z)$ is an entire function, and its derivatives are given by term-by-term differentiation of the series.

In the power expansion of $f(z)$ about the origin each term $a_{n} p_{n}(z)$ contributes by ( 1 ) distinct powers of the variable, and at least the greatest of these has non-zero coefficient. Thus the expansion has infinitely many non-zero terms and is not the expansion of a polynomial. Thus $f(z)$ is a transcendental entire function.

Every algebraic number $\zeta$ is a $(k+1)$-tuple zero of at least one polynomial in the sequence $Q$ (indeed of infinitely many polynomials). Thus for every algebraic number $\zeta$ and every non-negative rational integer $k$ there is a positive rational integer $N=N(k, \zeta)$ such that the polynomial $q_{N+1}^{(k)}(\zeta)$ vanishes.

But then the polynomials

Hence

$$
p_{N+1}^{(k)}(\zeta), p_{N+2}^{(k)}(\zeta), \cdots \text { all vanish. }
$$

$$
f^{(k)}(\zeta)=\sum_{n=1}^{\infty} a_{n} p_{n}^{(k)}(\zeta)=\sum_{n=1}^{N} a_{n} p_{n}^{(k)}(\zeta)
$$

But $\sum_{n=1}^{N} a_{n} p_{n}^{(k)}(z)$ is a polynomial with rational coefficients whence $f^{(k)}(\zeta)$ is an element of the field $R(\zeta)$ generated by $\zeta$ and the field $R$ of rational numbers.

Thus $f(z)$ and all its derivatives $f^{(k)}(z)(k=1,2, \cdots)$ are transcendental entire functions mapping every algebraic number field $K=R(\zeta)$ into itself.

\section{References}

[1] T. Schneider, Mathematische Annalen 121, (1949), 131-140.

[2] B. H. Neumann and R. Rado 'Monotone functions mapping the set of Rational Numbers onto itself', J. Aust. Math. Soc. 3 (1963), 281-287.

School of Mathematics

University of New South Wales 